\title{
Existence of Nonnegative Solutions of Nonlinear Fractional Parabolic Inequalities
}

\author{
Steven D. Taliaferro \\ Mathematics Department \\ Texas A\&M University \\ College Station, TX 77843
}

\begin{abstract}
We study the existence of nontrivial nonlocal nonnegative solutions $u(x, t)$ of the nonlinear initial value problems

$$
\begin{cases}\left(\partial_{t}-\Delta\right)^{\alpha} u \geq u^{\lambda} & \text { in } \mathbb{R}^{n} \times \mathbb{R}, n \geq 1 \\ u=0 & \text { in } \mathbb{R}^{n} \times(-\infty, 0)\end{cases}
$$

and

$$
\begin{cases}C_{1} u^{\lambda} \leq\left(\partial_{t}-\Delta\right)^{\alpha} u \leq C_{2} u^{\lambda} & \text { in } \mathbb{R}^{n} \times \mathbb{R}, n \geq 1 \\ u=0 & \text { in } \mathbb{R}^{n} \times(-\infty, 0),\end{cases}
$$

where $\lambda, \alpha, C_{1}$, and $C_{2}$ are positive constants with $C_{1}<C_{2}$. We use the definition of the fractional heat operator $\left(\partial_{t}-\Delta\right)^{\alpha}$ given in 31] and compare our results in the classical case $\alpha=1$ to known results.

2010 Mathematics Subject Classification. 35B09, 35B33, 35K58, 35R11.

Keywords. Fully fractional heat operator, Nonlocal solution.
\end{abstract}

\section{Introduction}

In this paper we study the existence of nontrivial nonlocal nonnegative solutions $u(x, t)$ of the nonlinear initial value problems

$$
\begin{cases}\left(\partial_{t}-\Delta\right)^{\alpha} u \geq u^{\lambda} & \text { in } \mathbb{R}^{n} \times \mathbb{R}, n \geq 1 \\ u=0 & \text { in } \mathbb{R}^{n} \times(-\infty, 0)\end{cases}
$$

and

$$
\begin{cases}C_{1} u^{\lambda} \leq\left(\partial_{t}-\Delta\right)^{\alpha} u \leq C_{2} u^{\lambda} & \text { in } \mathbb{R}^{n} \times \mathbb{R}, n \geq 1 \\ u=0 & \text { in } \mathbb{R}^{n} \times(-\infty, 0),\end{cases}
$$

where $\lambda, \alpha, C_{1}$, and $C_{2}$ are positive constants with $C_{1}<C_{2}$.

For a discussion of where the nonlocal fractional heat operator $\left(\partial_{t}-\Delta\right)^{\alpha}, \alpha>0$, arises naturally in applications, please see [23, 28].

For each of the problems (1.1), (1.2) and (1.3), (1.4), we compare our results in the classical case $\alpha=1$ to known results. Specifically, our result Theorem 2.1 in Section 2.1 for the problem (1.1), (1.2) implies 
(i) when $\lambda>1$ the existence of a critical exponent $\lambda_{0}(n, \alpha)>1$ for the nonexistence of nontrivial nonnegative solutions of the problem (1.1), (1.2), which agrees with the well-known Fujita exponent $\lambda_{F}=1+2 / n$ when $\alpha=1$ (see Remark 2.1) and

(ii) when $0<\lambda<1$ a nonexistence result for nontrivial nonnegative solutions of the problem (1.1), (1.2) which when $\alpha=1$ is similar to a result in 2] (see Section 2.11).

Similarly, our result Theorem 2.2 in Section 2.2 for the problem (1.3), (1.4) implies when $\lambda>1$ the existence of a critical exponent $\lambda_{1}(n, \alpha)>1$ for the existence of nontrivial nonnegative solutions of the problem (1.3), (1.4) which agrees when $\alpha=1$ with a critical exponent in [16] for the existence of self-similar solutions of the problem

$$
\begin{cases}\left(\partial_{t}-\Delta\right)^{\alpha} u=u^{\lambda} & \text { in } \mathbb{R}^{n} \times \mathbb{R}, n \geq 1 \\ u=0 & \text { in } \mathbb{R}^{n} \times(-\infty, 0)\end{cases}
$$

when $\alpha=1$ (see Section 2.2).

In order to complement our results for the two problems (1.1), (1.2) and (1.3), (1.4), we recall in Section 2.3 our result in 31 dealing with the existence of nontrivial nonlocal nonnegative solutions of the initial value problem

$$
\begin{cases}0 \leq\left(\partial_{t}-\Delta\right)^{\alpha} u \leq u^{\lambda} & \text { in } \mathbb{R}^{n} \times \mathbb{R}, n \geq 1 \\ u=0 & \text { in } \mathbb{R}^{n} \times(-\infty, 0) .\end{cases}
$$

We refer to the four problems (1.1, 1.2), (1.3, 1.4), (1.5, 1.6), and (1.7, 1.8) as the super problem, approximate problem, exact problem, and sub problem, respectively.

As in [31, we define the fully fractional nonlocal heat operator

$$
\left(\partial_{t}-\Delta\right)^{\alpha}: Y_{\alpha}^{p} \rightarrow X^{p}
$$

for

$$
\left(p>1 \text { and } 0<\alpha<\frac{n+2}{2 p}\right) \quad \text { or } \quad\left(p=1 \text { and } 0<\alpha \leq \frac{n+2}{2 p}\right)
$$

as the inverse of the operator

$$
J_{\alpha}: X^{p} \rightarrow Y_{\alpha}^{p}
$$

where

$$
\begin{gathered}
X^{p}:=\bigcap_{T \in \mathbb{R}} L^{p}\left(\mathbb{R}^{n} \times \mathbb{R}_{T}\right), \quad \mathbb{R}_{T}:=(-\infty, T), \\
J_{\alpha} f(x, t):=\iint_{\mathbb{R}^{n} \times \mathbb{R}_{t}} \Phi_{\alpha}(x-\xi, t-\tau) f(\xi, \tau) d \xi d \tau
\end{gathered}
$$

and

$$
Y_{\alpha}^{p}:=J_{\alpha}\left(X^{p}\right) .
$$

By (1.12) we mean $X^{p}$ is the set of all measurable functions $f: \mathbb{R}^{n} \times \mathbb{R} \rightarrow \mathbb{R}$ such that

$$
\|f\|_{L^{p}\left(\mathbb{R}^{n} \times \mathbb{R}_{T}\right)}<\infty \quad \text { for all } T \in \mathbb{R} .
$$

In the definition (1.13) of $J_{\alpha}$,

$$
\Phi_{\alpha}(x, t):=\frac{t^{\alpha-1}}{\Gamma(\alpha)} \frac{1}{(4 \pi t)^{n / 2}} e^{-|x|^{2} /(4 t)} \chi_{(0, \infty)}(t)
$$


is the fractional heat kernel.

When $p$ and $\alpha$ satisfy (1.10), it was shown in [31] that the operator (1.11) has the following properties:

(P1) it makes sense because $J_{\alpha} f \in L_{\mathrm{loc}}^{p}\left(\mathbb{R}^{n} \times \mathbb{R}\right)$ for $f \in X^{p}$,

(P2) it is one-to-one and onto, and

(P3) if $f \in X^{p}$ and $u=J_{\alpha} f$ then $f=0$ in $\mathbb{R}^{n} \times(-\infty, 0)$ if and only if $u=0$ in $\mathbb{R}^{n} \times(-\infty, 0)$.

By properties (P1) and (P2) we can indeed define (1.9) as the inverse of (1.11) when $p$ and $\alpha$ satisfy (1.10). Property (P3) will be needed to handle the initial condition $u=0$ in the above initial value problems.

Motivation for the above definition of (1.9) along with some more of its properties can be found in [31].

Stinga and Torrea [28] (see also Nyström and Sande [23]) gave an alternate definition of the fractional nonlocal heat operator

$$
\left(\partial_{t}-\Delta\right)^{\alpha}: U \rightarrow V
$$

which agrees with our definition (1.9) on the intersection $U \cap Y_{\alpha}^{p}$ of their domains. Functions $u: \mathbb{R}^{n} \times \mathbb{R} \rightarrow \mathbb{R}$ in $U$ are required to be bounded and sufficiently smooth. Their definition, unlike ours, is well suited for studying the Dirichlet problem for

$$
\left(\partial_{t}-\Delta\right)^{\alpha} u=f \quad \text { in } \Omega \times(0, T)
$$

where $\Omega \subset \mathbb{R}^{n}$ is a bounded domain. However our definition seems more suited for studying (1.16) when $\Omega=\mathbb{R}^{n}$ and $T=\infty$ because functions in $Y_{\alpha}^{p}$ can be discontinuous and locally unbounded, which allows for a greater variety of solutions of (1.16).

The operator (1.9) is a fully fractional heat operator as opposed to time fractional heat operators in which the fractional derivatives are only with respect to $t$, and space fractional heat operators, in which the fractional derivatives are only with respect to $x$.

Some recent results for nonlinear PDEs containing time (resp. space) fractional heat operators can be found in [3, 5, 6, 11, 18, 19, 20, 24, 29, 35, 36] (resp. [1, 4, 8, 9, 10, 14, 15, 17, 22, 25, 30, 32, 33, 34]). Except for [31, we know of no results for nonlinear PDEs containing the fully fractional heat operator $\left(\partial_{t}-\Delta\right)^{\alpha}$. However results for linear PDEs containing this operator, including in particular

$$
\left(\partial_{t}-\Delta\right)^{\alpha} u=f
$$

where $f$ is a given function, can be found in [7, 23, 27, 28].

\section{Statement and relevance of results}

In this section we state our results and relate them to results in [2, 13, 16, 31. In order to do this, we first note that for each fixed $p \geq 1$ the open first quadrant of the $\lambda \alpha$-plane is the union of 


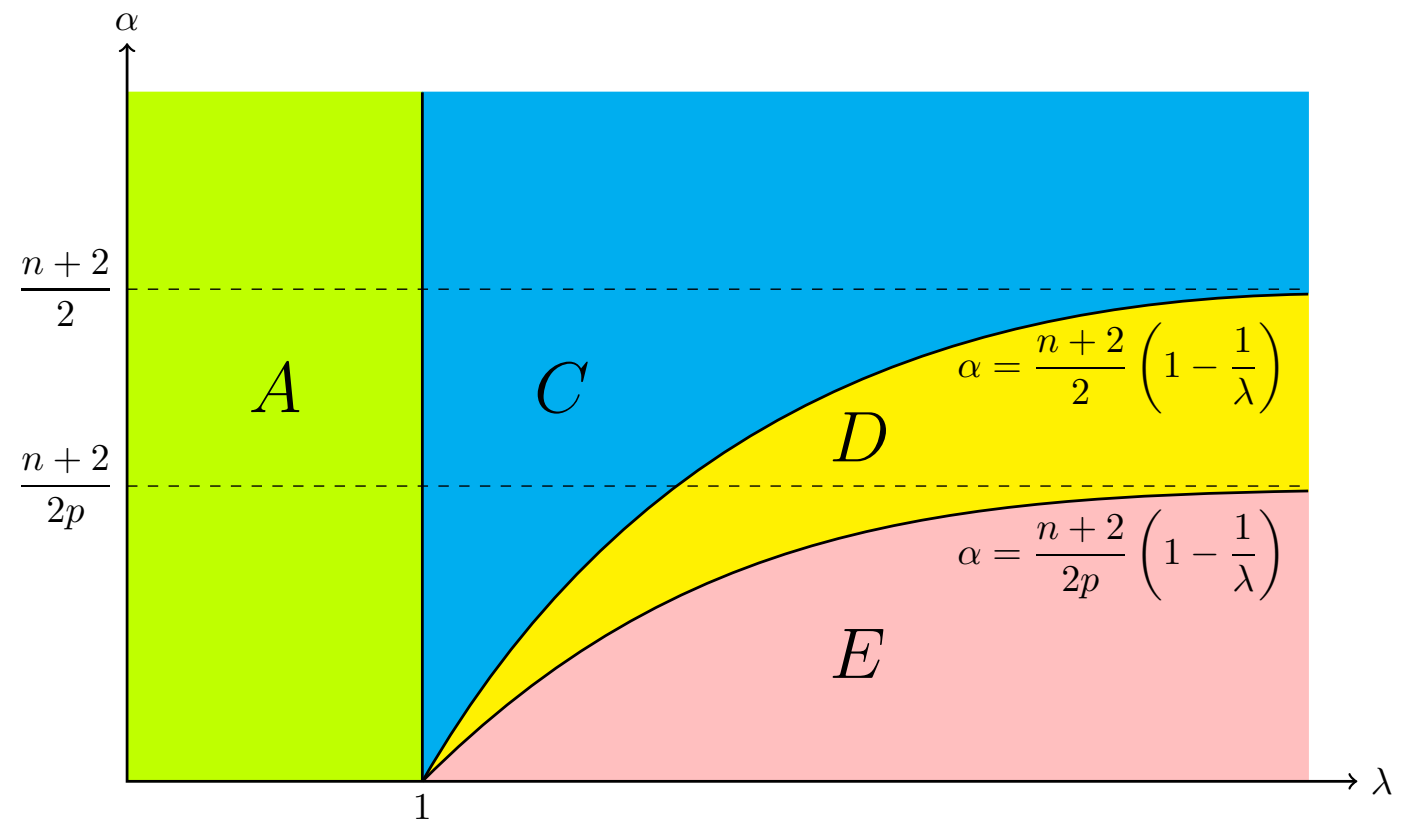

Figure 1: Graphs of the sets $A, B, C, D$, and $E$.

the following pairwise disjoint sets which are graphed in Figure 1;

$$
\begin{aligned}
& A:=\{(\lambda, \alpha): 0<\lambda<1 \text { and } \alpha>0\} \\
& B:=\{(\lambda, \alpha): \lambda=1 \text { and } \alpha>0\} \\
& C:=\left\{(\lambda, \alpha): \lambda>1 \text { and } \alpha \geq \frac{n+2}{2}\left(1-\frac{1}{\lambda}\right)\right\} \\
& D:=\left\{(\lambda, \alpha): \lambda>1 \text { and } \frac{n+2}{2 p}\left(1-\frac{1}{\lambda}\right) \leq \alpha<\frac{n+2}{2}\left(1-\frac{1}{\lambda}\right)\right\}, \\
& E:=\left\{(\lambda, \alpha): \lambda>1 \text { and } 0<\alpha<\frac{n+2}{2 p}\left(1-\frac{1}{\lambda}\right)\right\} .
\end{aligned}
$$

Note that if $p=1$ then $D=\emptyset$.

\subsection{The super problem}

Our result for the super problem (1.1), (1.2) is the following.

Theorem 2.1. Suppose $\alpha$ and $p$ satisfy (1.10) and $\lambda>0$. Then the super problem (1.1), (1.2) has a nontrivial nonnegative solution $u \in Y_{\alpha}^{p}$ if and only if

$$
(\lambda, \alpha) \in B \cup D \cup E .
$$

An immediate consequence of Theorem 2.1 is the following corollary.

Corollary 2.1. Suppose $\alpha$ and p satisfy (1.10) and $0<\lambda<1$. Then the only nonnegative solution $u \in Y_{\alpha}^{p}$ of the super problem (1.1), (1.2) is the trivial solution $u \equiv 0$. 
A result similar to Corollary 2.1 when $\alpha=1$ was proved in [2] for mild nonnegative super solutions of the initial value problem

$$
\begin{array}{ll}
\left(\partial_{t}-\Delta\right) u=u^{\lambda} & \text { in } \mathbb{R}^{n} \times(0, \infty) \\
u(x, 0)=u_{0}(x) & \text { for } x \in \mathbb{R}^{n},
\end{array}
$$

where $0<\lambda<1$.

Since (1.10) holds when $p=1$ and $0<\alpha \leq(n+2) / 2$ and since $(\lambda, \alpha) \in D \cup E$ if and only if

$$
0<\alpha<\frac{n+2}{2} \text { and } \lambda>\lambda_{0}(n, \alpha):=1+\frac{2 \alpha}{n+2-2 \alpha}
$$

(see Figure 1), we obtain also from Theorem 2.1 the following result.

Corollary 2.2. Suppose $0<\alpha \leq(n+2) / 2$ and $\lambda>1$. Then the super problem (1.1), (1.2) has a nontrivial nonnegative solution $u \in Y_{\alpha}^{1}$ if and only if

$$
\alpha \neq(n+2) / 2 \quad \text { and } \quad \lambda>\lambda_{0}(n, \alpha) .
$$

For comparison with Corollary 2.2, we recall the famous Fujita result [13], the following improved version of which appears in [26, Theorem 18.1].

Theorem A. If $1<\lambda \leq 1+2 / n$ then the only nonnegative solution $u \in L_{\text {loc }}^{\lambda}\left(\mathbb{R}^{n} \times(0, \infty)\right)$ of the inequality

$$
\left(\partial_{t}-\Delta\right) u \geq u^{\lambda} \quad \text { in } \mathcal{D}^{\prime}\left(\mathbb{R}^{n} \times(0, \infty)\right)
$$

is the trivial solution $u \equiv 0$.

Remark 2.1. Since $\lambda_{0}(n, 1)=1+2 / n$, we see that Corollary 2.2 can be viewed as a fractional nonlocal version of Theorem $\mathrm{A}$ and $\lambda_{0}(n, \alpha)$ for $0<\alpha<(n+2) / 2$ can be viewed as the critical exponent of Fujita type for nonnegative solutions $u \in Y_{\alpha}^{1}$ of the super problem (1.1), (1.2).

For the proof of Theorem 2.1 we will need the following lemma of independent interest which gives in particular conditions for the nonexistence of nontrivial nonnegative solutions of

$$
\left(\partial_{t}-\Delta\right)^{m} u \geq u^{\lambda} \quad \text { in } \mathcal{D}^{\prime}\left(\mathbb{R}^{n} \times(0, \infty)\right)
$$

where $m$ is a positive integer.

Lemma 2.1. Suppose $m$ is a positive integer,

$$
K>0, \quad(\lambda, \alpha) \in C, \quad \alpha \leq m,
$$

and $u \in L_{\text {loc }}^{\lambda}\left(\mathbb{R}^{n} \times(0, \infty)\right)$ is a nonnegative solution of

$$
\left(\partial_{t}-\Delta\right)^{m} u \geq\left(K(t+1)^{-(m-\alpha)} u\right)^{\lambda} \quad \text { in } \mathcal{D}^{\prime}\left(\mathbb{R}^{n} \times(0, \infty)\right)
$$

such that

$$
\left(\partial_{t}-\Delta\right)^{j} u \in L_{l o c}^{1}\left(\mathbb{R}^{n} \times(0, \infty)\right), \quad j=1,2, \ldots, m-1
$$

and

$$
\left(\partial_{t}-\Delta\right)^{j} u \geq 0, \quad j=1,2, \ldots, m-1
$$

Then

$$
u=0 \quad \text { in } \mathcal{D}^{\prime}\left(\mathbb{R}^{n} \times(0, \infty)\right) .
$$

Remark 2.2. Since $(\lambda, 1) \in C$ if and only if $1<\lambda \leq 1+2 / n$, we see that a consequence of Lemma 2.1 with $m=\alpha=K=1$ is Theorem A, 


\subsection{The approximate and exact problems}

If $u \in Y_{\alpha}^{p}$, where $\alpha$ and $p$ satisfy (1.10), is a solution of the exact problem (1.5), (1.6) then for all $\beta>0$ so is

$$
u_{\beta}(x, t):=\beta^{-\frac{2 \alpha}{\lambda-1}} u\left(x / \beta, t / \beta^{2}\right) .
$$

If, in addition, $u$ is self-similar, that is $u_{\beta}=u$ for all $\beta>0$, then substituting $\beta=\sqrt{t}, t>0$, in (2.6), we see that

$$
u(x, t)= \begin{cases}t^{-\frac{\alpha}{\lambda-1}} u(x / \sqrt{t}, 1) & \text { for }(x, t) \in \mathbb{R}^{n} \times(0, \infty) \\ 0 & \text { for }(x, t) \in \mathbb{R}^{n} \times(-\infty, 0] .\end{cases}
$$

Moreover, any function $u$ satisfying (2.7) is self-similar. Inspired by [16], we will seek in this section solutions of the approximate problem (1.3), (1.4) of the form (2.7).

We have no results for solutions $u \in Y_{\alpha}^{p}$ of the approximate problem (1.3), (1.4) when $p>1$ and $(\lambda, \alpha)$ lies in the curve

$$
\alpha=\frac{n+2}{2 p}\left(1-\frac{1}{\lambda}\right), \quad 1<\lambda<\infty
$$

which is graphed in Figure 1, Otherwise we have the following result.

Theorem 2.2. Suppose $\lambda>0$ and either

(i) $\alpha$ and $p$ satisfy $(1.10)_{2}$, or

(ii) $\alpha$ and $p$ satisfy $(1.10)$ and the point $(\lambda, \alpha)$ does not lie on the curve (2.8).

Then there exist positive constants $C_{1}$ and $C_{2}$ such that the approximate problem (1.3), (1.4) has a nontrivial nonnegative solution $u \in Y_{\alpha}^{p}$ if and only if

$$
(\lambda, \alpha) \in E .
$$

In this case, such a solution is given by

$$
u(x, t)= \begin{cases}t^{-\frac{\alpha}{\lambda-1}} w_{\alpha}(x / \sqrt{t}) & \text { for }(x, t) \in \mathbb{R}^{n} \times(0, \infty) \\ 0 & \text { for }(x, t) \in \mathbb{R}^{n} \times(-\infty, 0]\end{cases}
$$

where

$$
w_{\alpha}(z)=e^{-\frac{|z|^{2}}{4}}\left(|z|^{2}+1\right)^{-\left(\frac{n+2}{2}-\frac{\alpha \lambda}{\lambda-1}\right)} \quad \text { for } z \in \mathbb{R}^{n}
$$

An immediate consequence of Theorem 2.2 is the following corollary.

Corollary 2.3. Suppose

$$
p \geq 1, \quad 0<\alpha<\frac{n+2}{2 p}, \quad \text { and } \quad \lambda>\frac{n+2}{n+2-2 p \alpha} .
$$

Then there exist positive constants $C_{1}$ and $C_{2}$ such that a nontrivial nonnegative solution $u \in Y_{\alpha}^{p}$ of the approximate problem (1.3), (1.4) is given by (2.9) where $w_{\alpha}$ is defined in (2.10).

Since the conditions (2.11) hold if

$$
\alpha=1, \quad 1 \leq p<\frac{n+2}{2}, \quad \text { and } \quad \lambda>\frac{n+2}{n+2-2 p},
$$

we see that Corollary 2.3 can be viewed as an $\alpha \neq 1$ version of the following $\alpha=1$ result in [16] for the exact problem (1.5), (1.6). 
Theorem B ([16]). Suppose

$$
1 \leq p<\frac{n+2}{2} \quad \text { and } \quad \frac{n+2}{n+2-2 p}<\lambda< \begin{cases}\frac{n+2}{n-2} & \text { if } n \geq 3 \\ \infty & \text { if } n=1 \text { or } 2 .\end{cases}
$$

Then a nontrivial nonnegative solution $u \in Y_{1}^{p}$ of the exact problem (1.5), (1.6) with $\alpha=1$ is given by

$$
u(x, t)= \begin{cases}t^{-\frac{1}{\lambda-1}} w(x / \sqrt{t}) & \text { for }(x, t) \in \mathbb{R}^{n} \times(0, \infty) \\ 0 & \text { for }(x, t) \in \mathbb{R}^{n} \times(-\infty, 0]\end{cases}
$$

for some positive radial function $w: \mathbb{R}^{n} \rightarrow \mathbb{R}$ such that

$$
\frac{w(z)}{w_{1}(z)} \quad \text { is bounded between positive constants on } \mathbb{R}^{n}
$$

where $w_{\alpha}$ is defined in (2.10).

We have no results for the exact problem, but Corollary 2.3 and Theorem $\mathrm{B}$ motivate the following open question.

Open question. Suppose $\alpha$ and $p$ satisfy (1.10) so that the operator (1.9) is defined. For what $\lambda>0$ does there exist a nontrivial nonnegative solution $u \in Y_{\alpha}^{p}$ of the exact problem (1.5), (1.6)?

Since any solution of the exact problem (1.5), (1.6) is also a solution of the approximate problem (1.3), (1.4), it follows from Theorem 2.2 that a necessary condition on $\lambda$ is that either $p>1$ and $(\lambda, \alpha)$ lies on the curve (2.8) or $(\lambda, \alpha) \in E$ (see Figure 1).

\subsection{The sub problem}

We have no results for solutions $u \in Y_{\alpha}^{p}$ of the sub problem (1.7), (1.8) when $p \geq 1$ and the point $(\lambda, \alpha)$ lies on the curve (2.8) . Otherwise we have the following result from [31.

Theorem 2.3. Suppose $\alpha$ and $p$ satisfy (1.10), $\lambda>0$, and the point $(\lambda, \alpha)$ does not lie on the curve (2.8). Then the sub problem (1.7), (1.8) has a nontrivial nonnegative solution $u \in Y_{\alpha}^{p}$ if and only if

$$
(\lambda, \alpha) \in A \cup E .
$$

\section{$3 \quad J_{\alpha}$ version of results}

We define the $J_{\alpha}$ versions of the super, approximate, exact, and sub problems in Section 1 to be respectively the problems

$$
\begin{gathered}
\begin{cases}f \geq\left(J_{\alpha} f\right)^{\lambda} & \text { in } \mathbb{R}^{n} \times \mathbb{R} \\
f=0 & \text { in } \mathbb{R}^{n} \times(-\infty, 0) ;\end{cases} \\
\begin{cases}C_{1} f \leq\left(J_{\alpha} f\right)^{\lambda} \leq C_{2} f & \text { in } \mathbb{R}^{n} \times \mathbb{R} \\
f=0 & \text { in } \mathbb{R}^{n} \times(-\infty, 0) ;\end{cases}
\end{gathered}
$$




$$
\begin{cases}f=\left(J_{\alpha} f\right)^{\lambda} & \text { in } \mathbb{R}^{n} \times \mathbb{R} \\ f=0 & \text { in } \mathbb{R}^{n} \times(-\infty, 0)\end{cases}
$$

and

$$
\begin{cases}0 \leq f \leq\left(J_{\alpha} f\right)^{\lambda} & \text { in } \mathbb{R}^{n} \times \mathbb{R} \\ f=0 & \text { in } \mathbb{R}^{n} \times(-\infty, 0) ;\end{cases}
$$

which we will refer to respectively as the super $J_{\alpha}$ problem, approximate $J_{\alpha}$ problem, exact $J_{\alpha}$ problem, and sub $J_{\alpha}$ problem (or collectively as the $J_{\alpha}$ problems).

If $\alpha$ and $p$ satisfy (1.10) and $\lambda>0$ then by properties (P1)-(P3) in Section 1 of $J_{\alpha}$ and the definition of the fractional heat operator (1.9), $u$ is a nonnegative solution in $Y_{\alpha}^{p}$ of the super (approximate, exact, sub) problem in Section 1 if and only if

$$
f:=\left(\partial_{t}-\Delta\right)^{\alpha} u
$$

is a nonnegative solution in $X^{p}$ of the super (approximate, exact, sub) $J_{\alpha}$ problem in this section. (However, the positive constants $C_{1}$ and $C_{2}$ in (1.3), (1.4) may be different than the positive constants $C_{1}$ and $C_{2}$ in (3.3), (3.4).)

Since we will only consider solutions $f$ of the $J_{\alpha}$ problems which are nonnegative on $\mathbb{R}^{n} \times \mathbb{R}$, $J_{\alpha} f$ in these problems will always be a well-defined nonnegative extended real valued function on $\mathbb{R}^{n} \times \mathbb{R}$ even when the condition (1.10) is replace with the weaker condition that

$$
p \in[1, \infty) \text { and } \quad \alpha>0 .
$$

Hence in this section we study the $J_{\alpha}$ problems with condition (1.10) replaced with (3.9). However our results in this section for the $J_{\alpha}$ problems will only yield corresponding results for the original versions of these problems in Section 1 when (1.10) holds, for otherwise the fractional heat operators in these original problems are not defined. (For a more detailed discussion of the properties of $J_{\alpha}$ when (1.10) does not hold see [31, Section 4].)

Under the equivalence discussed above of the $J_{\alpha}$ problems and original versions of these problems in Section 1, the following Theorems 3.1 3.3, when restricted to the case that (1.10) holds, clearly imply Theorem 2.12 .3 respectively.

Theorem 3.1. Suppose $p$ and $\alpha$ satisfy (3.9) and $\lambda>0$. Then the super $J_{\alpha}$ problem (3.1), (3.2) has a nontrivial nonnegative solution $f \in X^{p}$ if and only if

$$
(\lambda, \alpha) \in B \cup D \cup E .
$$

Theorem 3.2. Suppose $\lambda, \alpha>0$ and either

(i) $p=1$ or

(ii) $p \in(1, \infty)$ and the point $(\lambda, \alpha)$ does not lie of the curve (2.8).

Then there exist positive constants $C_{1}$ and $C_{2}$ such that the approximate $J_{\alpha}$ problem (3.3), (3.4) has a nontrivial nonnegative solution $f \in X^{p}$ if and only if

$$
(\lambda, \alpha) \in E .
$$

In this case, such a solution is given by $f=u^{\lambda}$, where $u$ is defined by 2.9. 
Theorem 3.3. Suppose $\alpha$ and $p$ satisfy (3.9), $\lambda>0$, and the point $(\lambda, \alpha)$ does not lie on the curve (2.8). Then the sub $J_{\alpha}$ problem (3.7), (3.8) has a nontrivial nonnegative solution $f \in X^{p}$ if and only if

$$
(\lambda, \alpha) \in A \cup E .
$$

We will prove Theorems 3.1 and 3.2 in Section 5. We proved Theorem 3.3 in [31].

\section{Preliminary results}

In this section we provide some remarks and lemmas needed for the proofs of our results in Section 3 dealing with solutions of the super $J_{\alpha}$ problem (3.1), (3.2) and the approximate $J_{\alpha}$ problem (3.3), (3.4).

Lemma 4.1. Suppose $\alpha, \beta \in(0, \infty), x \in \mathbb{R}^{n}$, and $0<\tau<t$. Then

$$
\int_{\mathbb{R}^{n}} \Phi_{\alpha}(x-\xi, t-\tau) \Phi_{\beta}(\xi, \tau) d \xi=\frac{(t-\tau)^{\alpha-1} \tau^{\beta-1}}{\Gamma(\alpha) \Gamma(\beta)} \Phi_{1}(x, t) .
$$

Proof. Denote the left side of (4.1) by $h(x, t, \tau)$. Using the convolution theorem and the well-known fact that the Fourier transform with respect to $x$ of $\Phi_{\alpha}(x, t)$ is given by

$$
\hat{\Phi}_{\alpha}(\cdot, t)(y)=\frac{t^{\alpha-1}}{\Gamma(\alpha)} e^{-t|y|^{2}} \quad \text { for } t>0 \text { and } y \in \mathbb{R}^{n}
$$

we find for $0<\tau<t$ that

$$
\begin{aligned}
\hat{h}(\cdot, t, \tau)(y) & =\left(\frac{(t-\tau)^{\alpha-1}}{\Gamma(\alpha)} e^{-(t-\tau)|y|^{2}}\right)\left(\frac{\tau^{\beta-1}}{\Gamma(\beta)} e^{-\tau|y|^{2}}\right) \\
& =\frac{(t-\tau)^{\alpha-1} \tau^{\beta-1}}{\Gamma(\alpha) \Gamma(\beta)} e^{-t|y|^{2}} \\
& =\frac{(t-\tau)^{\alpha-1} \tau^{\beta-1}}{\Gamma(\alpha) \Gamma(\beta)} \hat{\Phi}_{1}(\cdot, t)(y)
\end{aligned}
$$

which proves (4.1).

Lemma 4.2. Suppose $\lambda, \alpha, T \in(0, \infty), p \in[1, \infty]$, and

$$
f(x, t)=g(x, t+T) \chi_{[0, \infty)}(t) \quad \text { for }(x, t) \in \mathbb{R}^{n} \times \mathbb{R}
$$

where $g: \mathbb{R}^{n} \times \mathbb{R} \rightarrow[0, \infty)$ is a measurable function such that

$$
\|g\|_{L^{p}\left(\mathbb{R}^{n} \times(T, \hat{T})\right)}<\infty \quad \text { for all } \hat{T}>T
$$

and

$$
g \geq\left(J_{\alpha} g\right)^{\lambda} \quad \text { in } \mathbb{R}^{n} \times \mathbb{R} .
$$

Then $f \in X^{p}$ and $f$ is a solution of the super $J_{\alpha}$ problem (3.1), (3.2).

Proof. For $t>0$ we have

$$
\begin{aligned}
\|f\|_{L^{p}\left(\mathbb{R}^{n} \times(-\infty, t)\right)} & =\|f\|_{L^{p}\left(\mathbb{R}^{n} \times(0, t)\right)} \\
& =\|g\|_{L^{p}\left(\mathbb{R}^{n} \times(T, t+T)\right)}<\infty
\end{aligned}
$$


by (4.3). Thus $f \in X^{p}$.

Clearly $f$ satisfies (3.2). Since (3.1) clearly holds in $\mathbb{R}^{n} \times(-\infty, 0]$, it remains only to prove (3.1) holds in $\mathbb{R}^{n} \times(0, \infty)$.

For $(x, t) \in \mathbb{R}^{n} \times(0, \infty)$ we find from (4.4) that

$$
\begin{aligned}
\left(J_{\alpha} f\right)(x, t) & =\int_{0}^{t} \int_{\mathbb{R}^{n}} \Phi_{\alpha}(x-\xi, t-\tau) g(\xi, \tau+T) d \xi d \tau \\
& =\int_{T}^{t} \int_{\mathbb{R}^{n}} \Phi_{\alpha}(x-\xi, \hat{t}-\hat{\tau}) g(\xi, \hat{\tau}) d \xi d \hat{\tau} \quad \text { where } \hat{t}=t+T \text { and } \hat{\tau}=\tau+T \\
& \leq \int_{-\infty}^{\hat{t}} \int_{\mathbb{R}^{n}} \Phi_{\alpha}(x-\xi, \hat{t}-\hat{\tau}) g(\xi, \hat{\tau}) d \xi d \hat{\tau} \\
& =\left(J_{\alpha} g\right)(x, \hat{t}) \\
& \leq g(x, \hat{t})^{1 / \lambda} \\
& =g(x, t+T)^{1 / \lambda}=f(x, t)^{1 / \lambda}
\end{aligned}
$$

Thus (3.1) holds in $\mathbb{R}^{n} \times(0, \infty)$.

Remark 4.1. Note for use in Lemma 4.2 that if

$$
0 \leq g(x, t) \leq \psi(t) \Phi_{\beta}(x, t) \quad \text { for }(x, t) \in \mathbb{R}^{n} \times \mathbb{R}
$$

where $\beta \in(0, \infty)$ and $\psi: \mathbb{R} \rightarrow[0, \infty)$ is a continuous function then for $0<T<\hat{T}<\infty$ we have

$$
\|g\|_{L^{\infty}\left(\mathbb{R}^{n} \times(T, \hat{T})\right)}<\infty
$$

and for $p \in[1, \infty)$

$$
\begin{aligned}
& \|g\|_{L^{p}\left(\mathbb{R}^{n} \times(T, \hat{T})\right)}^{p} \leq \int_{T}^{\hat{T}} \psi(t)^{p}\left(\frac{t^{\beta-1-n / 2}}{\Gamma(\beta)(4 \pi)^{n / 2}}\right)^{p}\left(\int_{\mathbb{R}^{n}} e^{\frac{-p|x|^{2}}{4 t}} d x\right) d t \\
& \leq(\hat{T}-T) \max _{T \leq t \leq \hat{T}} \psi(t)^{p}\left(\frac{t^{\beta-1-n / 2}}{\Gamma(\beta)(4 \pi)^{n / 2}}\right)^{p} \int_{\mathbb{R}^{n}} e^{\frac{-p|x|^{2}}{4 \hat{T}}} d x<\infty .
\end{aligned}
$$

Thus $g$ satisfies (4.3).

Our proof of Lemma 2.1 is a modification of [26, Proof of Theorem 18.1(i)] and in particular requires the following lemma. See [26, pages 101-102] for its proof.

Lemma 4.3. Suppose $v, f \in L_{l o c}^{1}\left(\mathbb{R}^{n} \times(0, \infty)\right)$ are nonnegative functions such that

$$
\left(\partial_{t}-\Delta\right) v \geq f \quad \text { in } \mathcal{D}^{\prime}\left(\mathbb{R}^{n} \times(0, \infty)\right)
$$

Let

$$
\varphi \in C_{0}^{\infty}\left(B_{1}(0)\right) \quad \text { and } \quad \psi \in C_{0}^{\infty}((-1,1))
$$

be nonnegative functions such that

$$
\varphi=1 \text { in } B_{1 / 2}(0), \quad \psi=1 \text { in }[0,1 / 2), \quad \text { and } \varphi, \psi \leq 1 .
$$


For $R>1, \beta>2$, and $t_{0}>0$ define

$$
\varphi_{R}(x)=\varphi\left(\frac{x}{R}\right)^{\beta} \quad \text { for } x \in \mathbb{R}^{n}
$$

and

$$
\psi_{R}(t)=\psi\left(\frac{t-t_{0}}{R^{2}}\right)^{\beta} \quad \text { for } t \geq t_{0} .
$$

Then

$$
\int_{t_{0}}^{\infty} \int_{\mathbb{R}^{n}} f \varphi_{R} \psi_{R} d x d t \leq \frac{C}{R^{2}} \iint_{Q_{R}} v\left(\varphi_{R} \psi_{R}\right)^{\frac{\beta-2}{\beta}} d x d t
$$

where $C>0$ does not depend on $R$ and

$$
Q_{R}=\left(B_{R}(0) \times\left(t_{0}, t_{0}+R^{2}\right)\right) \backslash\left(B_{R / 2}(0) \times\left(t_{0}, t_{0}+R^{2} / 2\right)\right) .
$$

Proof of Lemma 2.1. For $R>1, \gamma>2 m$, and $t_{0}>0$ define

$$
\varphi_{R}(x)=\varphi\left(\frac{x}{R}\right)^{\gamma} \quad \text { and } \quad \psi_{R}(t)=\psi\left(\frac{t-t_{0}}{R^{2}}\right)^{\gamma}
$$

for $x \in \mathbb{R}^{n}$ and $t \geq t_{0}$ where $\varphi$ and $\psi$ are as in Lemma 4.3. Let $f_{0}(x, t)$ be the function on the right side of (2.2).

For $j=1, \ldots, m$ we claim that

$$
\int_{t_{0}}^{\infty} \int_{\mathbb{R}^{n}} f_{0} \varphi_{R} \psi_{R} d x d t \leq\left(\frac{C}{R^{2}}\right)^{j} \iint_{Q_{R}}\left(H^{m-j} u\right)\left(\varphi_{R} \psi_{R}\right)^{\frac{\gamma-2 j}{\gamma}} d x d t
$$

where $H=\partial_{t}-\Delta, C>0$ does not depend on $R$, and $Q_{R}$ is defined in (4.5).

Inequality (4.6) holds for $j=1$ by (2.2) -(2.4) and Lemma 4.3 with $v=H^{m-1} u$ and $f=f_{0}$. Suppose inductively that (4.6) is true for some integer $j \in[1, m-1]$. Let

$$
\hat{\varphi}_{R}(x):=\varphi_{R}(x)^{\frac{\gamma-2 j}{\gamma}}=\varphi\left(\frac{x}{R}\right)^{\gamma-2 j} \quad \text { for } x \in \mathbb{R}^{n}
$$

and

$$
\hat{\psi}_{R}(t):=\psi_{R}(t)^{\frac{\gamma-2 j}{\gamma}}=\psi\left(\frac{t-t_{0}}{R^{2}}\right)^{\gamma-2 j} \text { for } t \geq t_{0} .
$$

Then using the inductive assumption, (2.3), (2.4), and Lemma 4.3 with

$$
f=H^{m-j} u, \quad v=H^{m-j-1} u, \quad \text { and } \quad \beta=\gamma-2 j,
$$

we find that

$$
\begin{gathered}
\int_{t_{0}}^{\infty} \int_{\mathbb{R}^{n}} f_{0} \varphi_{R} \psi_{R} d x d t \leq\left(\frac{C}{R^{2}}\right)^{j} \int_{t_{0}}^{\infty} \int_{\mathbb{R}^{n}}\left(H^{m-j} u\right) \hat{\varphi}_{R} \hat{\psi}_{R} d x d t \\
\leq\left(\frac{C}{R^{2}}\right)^{j}\left[\left(\frac{C}{R^{2}}\right) \iint_{Q_{R}}\left(H^{m-j-1} u\right)\left(\hat{\varphi}_{R} \hat{\psi}_{R}\right)^{\frac{\gamma-2 j-2}{\gamma-2 j}} d x d t\right] \\
=\left(\frac{C}{R^{2}}\right)^{j+1} \iint_{Q_{R}}\left(H^{m-(j+1)} u\right)\left(\varphi_{R} \psi_{R}\right)^{\frac{\gamma-2(j+1)}{\gamma}} d x d t
\end{gathered}
$$

which completes the inductive proof of (4.6) for $j=1,2, \ldots, m$. 
Taking $j=m$ in (4.6), defining $\gamma>2 m$ and $\lambda^{\prime}>1$ by

$$
\frac{2 m}{\gamma}=\frac{1}{\lambda^{\prime}}=1-\frac{1}{\lambda}
$$

and using Hölder's inequality, we have

$$
\begin{aligned}
\int_{t_{0}}^{\infty} \int_{\mathbb{R}^{n}} & \left((t+1)^{-(m-\alpha)} u\right)^{\lambda} \varphi_{R} \psi_{R} d x d t \\
& \leq \frac{C}{R^{2 m}} \iint_{Q_{R}} u\left(\varphi_{R} \psi_{R}\right)^{\frac{\gamma-2 m}{\gamma}=\frac{1}{\lambda}} \\
& =\frac{C}{R^{2 m}} \iint_{Q_{R}}(t+1)^{m-\alpha}(t+1)^{-(m-\alpha)} u\left(\varphi_{R} \psi_{R}\right)^{\frac{1}{\lambda}} d x d t \\
& \leq \frac{C}{R^{2 m}} I(R)\left(\iint_{Q_{R}}\left((t+1)^{-(m-\alpha)} u\right)^{\lambda} \varphi_{R} \psi_{R} d x d t\right)^{1 / \lambda}
\end{aligned}
$$

where

$$
\begin{aligned}
I(R) & =\left(\int_{t_{0}}^{t_{0}+R^{2}} \int_{|x|<R}(t+1)^{\lambda^{\prime}(m-\alpha)} d x d t\right)^{1 / \lambda^{\prime}} \\
& \leq C\left(R^{n+2} R^{2 \lambda^{\prime}(m-\alpha)}\right)^{1 / \lambda^{\prime}} \text { by (2.1) } \\
& =C R^{\frac{n+2}{\lambda^{\prime}}+2(m-\alpha)} .
\end{aligned}
$$

Hence

$$
\int_{t_{0}}^{\infty} \int_{\mathbb{R}^{n}}\left((t+1)^{-(m-\alpha)} u\right)^{\lambda} \varphi_{R} \psi_{R} d x d t \leq C R^{\frac{n+2}{\lambda^{\prime}}-2 \alpha}\left(\iint_{Q_{R}}\left((t+1)^{-(m-\alpha)} u\right)^{\lambda}\left(\varphi_{R} \psi_{R}\right) d x d t\right)^{1 / \lambda}
$$

which implies

$$
\int_{t_{0}}^{\infty} \int_{\mathbb{R}^{n}}\left((t+1)^{-(m-\alpha)} u\right)^{\lambda} \varphi_{R} \psi_{R} d x d t \leq C R^{\left(\frac{n+2}{\lambda^{\prime}}-2 \alpha\right) \lambda^{\prime}} .
$$

By (2.1), $2 \alpha \geq(n+2) / \lambda^{\prime}$. Hence, sending $R$ to $\infty$ in (4.8) we find that

$$
\int_{t_{0}}^{\infty} \int_{\mathbb{R}^{n}}\left((t+1)^{-(m-\alpha)} u\right)^{\lambda} d x d t<\infty
$$

which implies the integral on the right side of (4.7) tends to zero as $R \rightarrow \infty$. Thus sending $R$ to $\infty$ in (4.7) yields $u=0$ in $\mathbb{R}^{n} \times\left(t_{0}, \infty\right)$. Hence, since $t_{0}>0$ was arbitrary, we see that (2.5) holds.

Lemma 4.4. Suppose $p \in[1, \infty)$ and $f \in X^{p}$ is a nonnegative function satisfying (3.2). Then

$$
\begin{gathered}
J_{\alpha} f \in L_{l o c}^{1}\left(\mathbb{R}^{n} \times(0, \infty)\right) \quad \text { for } \alpha>0, \\
H J_{\alpha} f=J_{\alpha-1} f \quad \text { in } \mathcal{D}^{\prime}\left(\mathbb{R}^{n} \times(0, \infty)\right) \text { for } \alpha>1,
\end{gathered}
$$

and

$$
H J_{1} f=f \quad \text { in } \mathcal{D}^{\prime}\left(\mathbb{R}^{n} \times(0, \infty)\right)
$$

where $H=\partial_{t}-\Delta$. 
Proof. We will need the following easily-verified and/or well-known facts:

(i)

$$
\iint_{\mathbb{R}^{n} \times \mathbb{R}} \Phi_{1}(x-\xi, t-\tau) H^{*} \varphi(x, t) d x d t=\varphi(\xi, \tau)
$$

for $\varphi \in C_{0}^{\infty}\left(\mathbb{R}^{n} \times \mathbb{R}\right)$ where $H^{*}=\partial_{t}+\Delta$; and

(ii)

$$
\int_{0}^{b} \int_{B_{b}(0)} \Phi_{\alpha}(x-\xi, t-\tau) d x d t \in L^{q}\left(\mathbb{R}^{n} \times(0, b)\right)
$$

for $b, \alpha \in(0, \infty)$ and $q \in[1, \infty]$.

To prove (4.9), let $b, \alpha \in(0, \infty)$. Then by (4.13) and Hölder's inequality we have

$$
\begin{aligned}
& \int_{0}^{b} \int_{B_{b}(0)} J_{\alpha} f d x d t \\
& =\int_{0}^{b} \int_{B_{b}(0)} \int_{0}^{b} \int_{\mathbb{R}^{n}} \Phi_{\alpha}(x-\xi, t-\tau) f(\xi, \tau) d \xi d \tau d x d t \\
& =\int_{0}^{b} \int_{\mathbb{R}^{n}} f(\xi, \tau)\left(\int_{0}^{b} \int_{B_{b}(0)} \Phi_{\alpha}(x-\xi, t-\tau) d x d t\right) d \xi d \tau<\infty .
\end{aligned}
$$

Thus (4.9) holds.

To prove (4.10), suppose $\beta, \gamma \in(0, \infty), \beta+\gamma=\alpha>1$, and $\varphi \in C_{0}^{\infty}\left(\mathbb{R}^{n} \times(0, \infty)\right)$. Then assuming we can interchange the order of integration in the following calculation (we will justify this after the calculation) and using the fact that $\Phi_{\beta} * \Phi_{\gamma}=\Phi_{\alpha}$ (see [31, Lemma 5.1]), we find that

$$
\begin{aligned}
\left(H J_{\alpha} f\right) \varphi & =J_{\alpha} f\left(H^{*} \varphi\right)=\left(\Phi_{\alpha} * f\right)\left(H^{*} \varphi\right) \\
& =\left(\Phi_{\beta} * \Phi_{\gamma} * f\right)\left(H^{*} \varphi\right) \\
& =\iiint \int \Phi_{\gamma}(\eta-\xi, \zeta-\tau) f(\xi, \tau) \iint \Phi_{\beta}(x-\eta, t-\zeta) H^{*} \varphi(x, t) d x d t d \xi d \tau d \eta d \zeta \\
& =\iint J_{\gamma} f(\eta, \zeta)\left(\iint \Phi_{\beta}(x-\eta, t-\zeta) H^{*} \varphi(x, t) d x d t\right) d \eta d \zeta
\end{aligned}
$$

Taking $\beta=1$ and $\gamma=\alpha-1$ in (4.14) and using (4.12) we find that

$$
\left(H J_{\alpha} f\right) \varphi=\left(J_{\alpha-1} f\right)(\varphi) .
$$

Hence (4.10) holds provided we justify the calculation (4.14) by verifying

$$
\iint\left(\Phi_{\alpha} * f\right)\left|H^{*} \varphi\right|<\infty
$$

To do this, choose $b>0$ such that suppt $\varphi \subset B_{b}(0) \times(0, b)$ and repeat the calculation (4.14) with $\beta=\alpha-1$ and $\gamma=1$, and with $H^{*} \varphi$ replaced with $\left|H^{*} \varphi\right|$ to obtain

$$
\iint\left(\Phi_{\alpha} * f\right)\left|H^{*} \varphi\right|=\int_{0}^{b} \int_{\mathbb{R}^{n}} J_{1} f(\eta, \zeta)\left(\int_{0}^{b} \int_{B_{b}(0)} \Phi_{\alpha-1}(x-\eta, t-\zeta)\left|H^{*} \varphi(x, t)\right| d x d t\right) d \eta d \zeta .
$$


Choose $p^{\prime} \in(1, \infty)$ such that $0<\frac{1}{p}-\frac{1}{p^{\prime}}<\frac{2}{n+2}$. Then by [31, Lemma 7.2], $J_{1} f \in L^{p^{\prime}}\left(\mathbb{R}^{n} \times(0, b)\right)$. Hence (4.15) follows from (4.13), (4.16), and Hölder's inequality.

To prove (4.11), let $\varphi \in C_{0}^{\infty}\left(\mathbb{R}^{n} \times(0, \infty)\right)$. Then using (4.12) and assuming we can interchange the order of integration we see that

$$
\begin{aligned}
& H\left(J_{1} f\right) \varphi=\left(J_{1} f\right)\left(H^{*} \varphi\right)=\left(\Phi_{1} * f\right)\left(H^{*} \varphi\right) \\
& =\iint\left(\iint \Phi_{1}(x-\xi, t-\tau) H^{*} \varphi(x, t) d x d t\right) f(\xi, \tau) d \xi d \tau \\
& =f(\varphi)
\end{aligned}
$$

Thus (4.11) holds because interchanging the order of integration is validated by using (4.13) and Hölder's inequality as in the proof of (4.15).

Lemma 4.5 ([31], Lemma 7.4). Suppose $x \in \mathbb{R}^{n}$ and $t, \tau \in(0, \infty)$ satisfy

$$
|x|^{2}<t \quad \text { and } \quad \frac{t}{4}<\tau<\frac{3 t}{4} .
$$

Then

$$
\int_{|\xi|^{2}<\tau} \Phi_{1}(x-\xi, t-\tau) d \xi \geq C(n)>0
$$

where $\Phi_{\alpha}$ is defined by (1.15).

\section{Proof Theorems 3.1 and 3.2}

In this section we prove Theorems 3.1 and 3.2 .

Theorem 3.1 is a consequence of the following Theorems 5.1 5.4 because Theorem 5.1 (5.2, 5.3, 5.4) guarantees under the assumption on $p, \alpha$, and $\lambda$ in Theorem 3.1 the nonexistence (existence, nonexistence, existence) of nontrivial nonnegative solutions $f \in X^{p}$ of the super $J_{\alpha}$ problem (3.1), (3.2) when $(\lambda, \alpha) \in A(B, C, D \cup E)$.

Theorem 5.1. Suppose $f: \mathbb{R}^{n} \times \mathbb{R} \rightarrow[0, \infty)$ is a measurable solution of the super $J_{\alpha}$ problem (3.1), (3.2) where $\lambda \in(0,1)$ and $\alpha \in(0, \infty)$ are constants. Then either

$$
f=0 \quad \text { a.e. in } \mathbb{R}^{n} \times \mathbb{R}
$$

or there exists $a \in[0, \infty)$ such that

$$
\begin{gathered}
f=0 \quad \text { a.e. in } \mathbb{R}^{n} \times(-\infty, a), \\
f(x, t) \geq\left(M(t-a)^{\alpha}\right)^{\frac{\lambda}{1-\lambda}} \quad \text { a.e. in } \mathbb{R}^{n} \times(a, \infty),
\end{gathered}
$$

and

$$
\left(J_{\alpha} f\right)(x, t) \geq\left(M(t-a)^{\alpha}\right)^{\frac{1}{1-\lambda}} \quad \text { a.e. in } \mathbb{R}^{n} \times(a, \infty)
$$

where

$$
M=M(\lambda, \alpha)=\frac{\Gamma\left(\frac{\lambda \alpha}{1-\lambda}+1\right)}{\Gamma\left(\alpha+\frac{\lambda \alpha}{1-\lambda}+1\right)}
$$

where $\Gamma$ is the Gamma function. 
Proof. Let

$$
a=\sup \left\{t \in \mathbb{R}:\|f\|_{L^{\infty}\left(\mathbb{R}^{n} \times(-\infty, t)\right)}=0\right\} .
$$

Then (3.2) implies $a \geq 0$. If $a=\infty$ then (5.1) holds. Hence we can assume

$$
a \in[0, \infty) .
$$

It follows from (5.6) and (5.7) that (5.2) holds and it remains only to prove (5.3) and (5.4). To do this we first prove

$$
f(x, t) \geq\left(N_{0}(t-a)^{\alpha}\right)^{\frac{\lambda}{1-\lambda}} \quad \text { a.e. in } \mathbb{R}^{n} \times(a, \infty)
$$

for some positive constant $N_{0}=N_{0}(n, \lambda, \alpha)$. Let $T>0$ and $x_{0} \in \mathbb{R}^{n}$ be fixed. To prove (5.8) it suffices to prove

$$
f(x, t) \geq\left(N_{0}(t-a)^{\alpha}\right)^{\frac{\lambda}{1-\lambda}} \quad \text { for }(x, t) \in \Omega\left(x_{0}, a, T\right),
$$

and some positive constant $N_{0}=N_{0}(n, \lambda, \alpha)$ where

$$
\Omega\left(x_{0}, t_{0}, T\right):=\left\{(x, t) \in \mathbb{R}^{n} \times \mathbb{R}:\left|x-x_{0}\right|^{2}<\left(t-t_{0}\right)<T\right\}
$$

because

$$
\mathbb{R}^{n} \times(a, \infty)=\bigcup_{\substack{x_{0} \in \mathbb{R}^{n} \\ T>0}} \Omega\left(x_{0}, a, T\right)
$$

Let

$$
t_{0} \in(a, T+a)
$$

be fixed. Then to prove (5.9), and hence (5.8), it suffices to prove

$$
f(x, t) \geq\left(N_{0}\left(t-t_{0}\right)^{\alpha}\right)^{\frac{\lambda}{1-\lambda}} \quad \text { for }(x, t) \in \Omega\left(x_{0}, t_{0}, T\right)
$$

and some positive constant $N_{0}=N_{0}(n, \lambda, \alpha)$ because then sending $t_{0}$ to $a$ in (5.11) we get (5.9). Define

$$
g: \mathbb{R}^{n} \times \mathbb{R} \rightarrow[0, \infty)
$$

by

$$
g(x, t)=f\left(x+x_{0}, t+t_{0}\right) .
$$

Then $f$ satsifies (5.11), and hence (5.8), if and only if $g$ satsifies

$$
g(x, t) \geq\left(N_{0} t^{\alpha}\right)^{\frac{\lambda}{1-\lambda}} \quad \text { for }(x, t) \in \Omega(0,0, T) .
$$

It follows from (5.6) and (5.10) that $J_{\alpha} f$ is bounded below by a positive constant on bounded subsets of $\mathbb{R}^{n} \times\left(t_{0}, \infty\right)$, in particular on $\Omega\left(x_{0}, t_{0}, T\right)$. Hence by (3.1) and (5.12) we see that $g$ is bounded below by a positive constant on $\Omega(0,0, T)$. Thus there exists a constant $b_{0}>0$ such that

$$
g(x, t) \geq\left(b_{0} T^{\alpha}\right)^{\frac{\lambda}{1-\lambda}} \geq\left(b_{0} t^{\alpha}\right)^{\frac{\lambda}{1-\lambda}} \quad \text { for }(x, t) \in \Omega(0,0, T) .
$$

(Note however that $b_{0}$ may depend not only on $n, \lambda$, and $\alpha$ but also on $x_{0}, t_{0}$, and $T$.)

Also for $(x, t) \in \mathbb{R}^{n} \times \mathbb{R}$ we find from (5.12) and (3.1) that

$$
\begin{aligned}
g(x, t)^{1 / \lambda} & =f\left(x+x_{0}, t+t_{0}\right)^{1 / \lambda} \geq\left(J_{\alpha} f\right)\left(x+x_{0}, t+t_{0}\right) \\
& =\int_{-\infty}^{t+t_{0}} \int_{\mathbb{R}^{n}} \Phi_{\alpha}\left(x+x_{0}-\xi, t+t_{0}-\tau\right) f(\xi, \tau) d \xi d \tau \\
& =\int_{-\infty}^{t} \int_{\mathbb{R}^{n}} \Phi_{\alpha}(x-\bar{\xi}, t-\bar{\tau}) f\left(\bar{\xi}+x_{0}, \bar{\tau}+t_{0}\right) d \bar{\xi} d \bar{\tau} \\
& =J_{\alpha} g(x, t) .
\end{aligned}
$$


Thus

$$
g \geq\left(J_{\alpha} g\right)^{\lambda} \text { in } \mathbb{R}^{n} \times \mathbb{R} .
$$

Let $\beta:=\frac{\lambda \alpha}{1-\lambda}$. Then for $(x, t) \in \Omega(0,0, T)$ we obtain from (‥15), (5.14), and Lemma 4.5 that

$$
\begin{aligned}
& g(x, t)^{1 / \lambda} \geq J_{\alpha} g(x, t) \geq b_{0}^{\beta / \alpha} \iint_{\Omega(0,0, T)} \Phi_{\alpha}(x-\xi, t-\tau) \tau^{\beta} d \xi d \tau \\
& \geq b_{0}^{\beta / \alpha} \int_{t / 4}^{3 t / 4} \frac{(t-\tau)^{\alpha-1}}{\Gamma(\alpha)} \tau^{\beta}\left(\int_{|\xi|<\sqrt{\tau}} \Phi_{1}(x-\xi, t-\tau) d \xi\right) d \tau \\
& \geq b_{0}^{\beta / \alpha} C(n) \int_{t / 4}^{3 t / 4} \frac{(t-\tau)^{\alpha-1}}{\Gamma(\alpha)} \tau^{\beta} d \tau \\
& =b_{0}^{\beta / \alpha} \frac{C(n)}{\Gamma(\alpha)}\left(\int_{1 / 4}^{3 / 4}(1-s)^{\alpha-1} s^{\beta} d s\right) t^{\alpha+\beta=\frac{\alpha}{1-\lambda}} .
\end{aligned}
$$

Thus letting

$$
N_{0}=N_{0}(n, \lambda, \alpha)=\frac{C(n)}{\Gamma(\alpha)} \int_{1 / 4}^{3 / 4}(1-s)^{\alpha-1} s^{\frac{\lambda \alpha}{1-\lambda}} d s
$$

and $b_{1}=b_{0}^{\lambda} N_{0}^{1-\lambda}$ we have

$$
g(x, t) \geq\left(b_{1} t^{\alpha}\right)^{\frac{\lambda}{1-\lambda}} \quad \text { for }(x, t) \in \Omega(0,0, t)
$$

where

$$
\frac{b_{1}}{N_{0}}=\left(\frac{b_{0}}{N_{0}}\right)^{\lambda}
$$

Iterating the method we used to derive (5.16) from (5.14) we inductively obtain a sequence $\left\{b_{j}\right\}_{j=0}^{\infty} \subset(0, \infty)$ such that for $j=1,2, \ldots$ we have

$$
\frac{b_{j}}{N_{0}}=\left(\frac{b_{j-1}}{N_{0}}\right)^{\lambda}
$$

and

$$
g(x, t) \geq\left(b_{j} t^{\alpha}\right)^{\frac{\lambda}{1-\lambda}} \quad \text { for }(x, t) \in \Omega(0,0, T) .
$$

Since $\lambda \in(0,1)$ it follows from (5.17) that

$$
\lim _{j \rightarrow \infty} \frac{b_{j}}{N_{0}}=1 .
$$

Consequently, sending $j$ to $\infty$ in (5.18) we obtain (5.13) and hence also (5.8).

Using (3.1), (5.2), (5.8), and (5.5) and making the change of variables $\bar{t}=t-a, \bar{\tau}=\tau-a$ we obtain for $(x, t) \in \mathbb{R}^{n} \times(a, \infty)$ that

$$
\begin{aligned}
f(x, t)^{1 / \lambda} & \geq J_{\alpha} f(x, t) \\
& \geq N_{0}^{\frac{\lambda}{1-\lambda}} \int_{a}^{t} \frac{(t-\tau)^{\alpha-1}}{\Gamma(\alpha)}(\tau-a)^{\frac{\lambda \alpha}{1-\lambda}} d \tau \\
& =N_{0}^{\frac{\lambda}{1-\lambda}} \int_{0}^{\bar{t}} \frac{(\bar{t}-\bar{\tau})^{\alpha-1}}{\Gamma(\alpha)} \bar{\tau}^{\frac{\lambda \alpha}{1-\lambda}} d \bar{\tau} \\
& =N_{0}^{\frac{\lambda}{1-\lambda}} M \bar{t}^{\frac{\alpha}{1-\lambda}}=\left(N_{0}^{\lambda} M^{1-\lambda}(t-a)^{\alpha}\right)^{\frac{1}{1-\lambda}} .
\end{aligned}
$$


Thus for $(x, t) \in \mathbb{R}^{n} \times(a, \infty)$ we have

$$
f(x, t) \geq\left(J_{\alpha} f(x, t)\right)^{\lambda} \geq\left(N_{1}(t-a)^{\alpha}\right)^{\frac{\lambda}{1-\lambda}}
$$

where

$$
\frac{N_{1}}{M}=\left(\frac{N_{0}}{M}\right)^{\lambda}
$$

Iterating the method we used to derive (5.19) from (5.8) we inductively obtain a sequence $\left\{N_{j}\right\}_{j=0}^{\infty} \subset(0, \infty)$ such that for $j=1,2, \ldots$ we have

$$
\frac{N_{j}}{M}=\left(\frac{N_{j-1}}{M}\right)^{\lambda}
$$

and

$$
f(x, t) \geq\left(J_{\alpha} f(x, t)\right)^{\lambda} \geq\left(N_{j}(t-a)^{\alpha}\right)^{\frac{\lambda}{1-\lambda}} \text { for }(x, t) \in \mathbb{R}^{n} \times(a, \infty) .
$$

Since $\lambda \in(0,1)$ it follows from (5.20) that

$$
\lim _{j \rightarrow \infty} \frac{N_{j}}{M}=1
$$

Consequently, sending $j$ to $\infty$ in (5.21) we obtain (5.3) and (5.4).

Theorem 5.2. Suppose $\alpha, T \in(0, \infty)$ and $p \in[1, \infty]$. Then there exists $a=a(\alpha)>0$ such that $a$ solution

$$
f \in C^{\infty}\left(\mathbb{R}^{n} \times[0, \infty)\right) \cap X^{p}
$$

of the super $J_{\alpha}$ problem

$$
\begin{array}{ll}
f \geq J_{\alpha} f & \text { in } \mathbb{R}^{n} \times \mathbb{R} \\
f=0 & \text { in } \mathbb{R}^{n} \times(-\infty, 0)
\end{array}
$$

$i s$

$$
f(x, t)=e^{a(t+T)} \Phi_{1}(x, t+T) \chi_{[0, \infty)}(t) .
$$

Proof. For all $a>0$ the function $f$ given by (5.22) is in $C^{\infty}\left(\mathbb{R}^{n} \times[0, \infty)\right)$. Thus, to prove Theorem 5.2. it suffices by Lemma 4.2, to show there exists $a=a(\alpha)>0$ such that the function $g: \mathbb{R}^{n} \times \mathbb{R} \rightarrow$ $[0, \infty)$ defined by

$$
g(x, t)=e^{a t} \Phi_{1}(x, t)
$$

satisfies (4.3) and

$$
g \geq J_{\alpha} g \quad \text { in } \mathbb{R}^{n} \times \mathbb{R} .
$$

By Remark 4.1, $g$ satisfies (4.3) for all $a>0$. Hence it remains only to show there exists $a=a(\alpha)>0$ such that $g$ satisfies (5.24)

The inequality (5.24) holds in $\mathbb{R}^{n} \times(-\infty, 0]$ because $g=0$ there. On the other hand, for $(x, t) \in \mathbb{R}^{n} \times(0, \infty)$, it follows from (5.23) and Lemma 4.1 that

$$
\begin{aligned}
J_{\alpha} g(x, t) & =\int_{0}^{t}\left(\int_{\mathbb{R}^{n}} \Phi_{\alpha}(x-\xi, t-\tau) \Phi_{1}(\xi, \tau) d \xi\right) e^{a \tau} d \tau \\
& =\Phi_{1}(x, t) \int_{0}^{t} \frac{(t-\tau)^{\alpha-1}}{\Gamma(\alpha)} e^{a \tau} d \tau
\end{aligned}
$$


However, for $t, a>0$ we have

$$
\begin{gathered}
e^{-a t} \int_{0}^{t}(t-\tau)^{\alpha-1} e^{a \tau} d \tau \leq \int_{-\infty}^{t}(t-\tau)^{\alpha-1} e^{-a(t-\tau)} d \tau \\
=\frac{1}{a^{\alpha}} \int_{0}^{\infty} \zeta^{\alpha-1} e^{-\zeta} d \zeta \rightarrow 0 \quad \text { as } a \rightarrow \infty
\end{gathered}
$$

It follows therefore from (5.25) and (5.23) that there exists $a=a(\alpha)>0$ such that $g$ satisfies (5.24) in $\mathbb{R}^{n} \times(0, \infty)$.

Theorem 5.3. Suppose

$$
f \in X^{p}
$$

is a nonnegative solution of the super $J_{\alpha}$ problem (3.1), (3.2) where $p \in[1, \infty$ ) and

$$
(\lambda, \alpha) \in C
$$

where $C$ is the region defined in Section 2 and graphed in Figure 1. Then

$$
f=J_{\alpha} f=0 \quad \text { a.e. in } \mathbb{R}^{n} \times \mathbb{R} .
$$

Proof. By (3.1) and (3.2), $f$ satisfies (5.28) a.e. in $\mathbb{R}^{n} \times(-\infty, 0]$. Hence it suffices to prove

$$
f=J_{\alpha} f=0 \quad \text { a.e. in } \mathbb{R}^{n} \times(0, \infty) .
$$

Let $u=J_{m} f$ where $m$ is the positive integer satisfying $\alpha \leq m<\alpha+1$. By Lemma 4.4, $u$ satisfies (2.3) and (2.4) and

$$
\begin{aligned}
& H^{m} u(x, t)=f(x, t) \geq\left(J_{\alpha} f(x, t)\right)^{\lambda} \\
& =\left(\int_{0}^{t} \int_{\mathbb{R}^{n}} \frac{(t-\tau)^{(\alpha-m)+(m-1)}}{\Gamma(\alpha)} \Phi_{1}(x-\xi, t-\tau) f(\xi, \tau) d \xi d \tau\right)^{\lambda} \\
& \geq\left(\frac{\Gamma(m)}{\Gamma(\alpha)}(t+1)^{\alpha-m} J_{m} f(x, t)\right)^{\lambda}=\left(\frac{\Gamma(m)}{\Gamma(\alpha)}(t+1)^{\alpha-m} u(x, t)\right)^{\lambda} .
\end{aligned}
$$

Hence (5.29) follows from (5.27) and Lemma 2.1.

Theorem 5.4. Suppose

$$
\lambda>1, \quad 0<\alpha<\frac{n+2}{2}\left(1-\frac{1}{\lambda}\right), \quad p \in[1, \infty) \quad \text { and } T>0 .
$$

Then

$$
\beta:=\frac{n+2}{2}-\frac{\lambda \alpha}{\lambda-1}=\frac{n+2}{2}-\frac{\alpha}{1-\frac{1}{\lambda}}>0
$$

and a solution

$$
f \in C^{\infty}\left(\mathbb{R}^{n} \times[0, \infty)\right) \cap X^{p}
$$

of the super $J_{\alpha}$ problem (3.1), (3.2) is

$$
f(x, t)=A \Phi_{\beta}(x, t+T) \chi_{[0, \infty)}(t)
$$

where

$$
A=A(n, \lambda, \alpha)=\left(\frac{(4 \pi)^{(\lambda-1) n / 2} \Gamma(\alpha+\beta)^{\lambda}}{\Gamma(\beta)}\right)^{\frac{1}{\lambda-1}}
$$


Proof. Inequality (5.31) follows from (5.30) and $f$ given by (5.32) is clearly in $C^{\infty}\left(\mathbb{R}^{n} \times[0, \infty)\right)$. Thus, to prove Theorem [5.4, it suffices by Lemma 4.2 to show that the function $g: \mathbb{R}^{n} \times \mathbb{R} \rightarrow[0, \infty)$ defined by

$$
g(x, t)=A \Phi_{\beta}(x, t)
$$

satisfies (4.3) and (4.4).

By Remark 4.1, $g$ satisfies (4.3). Hence it remains only to show $g$ satsfies (4.4). The inequality (4.4) holds in $\mathbb{R}^{n} \times(-\infty, 0]$ because $g=0$ there. On the other hand, for $(x, t) \in \mathbb{R}^{n} \times(0, \infty)$, it follows from (5.31) and Lemma 4.1 that

$$
\begin{aligned}
J_{\alpha} g(x, t) & =A \int_{0}^{t}\left(\int_{\mathbb{R}^{n}} \Phi_{\alpha}(x-\xi, t-\tau) \Phi_{\beta}(\xi, \tau) d \xi\right) d \tau \\
& =A \Phi_{1}(x, t) \int_{0}^{t} \frac{(t-\tau)^{\alpha-1} \tau^{\beta-1}}{\Gamma(\alpha) \Gamma(\beta)} d \tau \\
& =A \Phi_{1}(x, t) \frac{t^{\alpha+\beta-1}}{\Gamma(\alpha+\beta)}=A \Phi_{\alpha+\beta}(x, t)
\end{aligned}
$$

and thus

$$
\begin{aligned}
\frac{\left(J_{\alpha} g(x, t)\right)^{\lambda}}{g(x, t)} & =A^{\lambda-1} \frac{\Gamma(\beta) t^{\lambda \alpha+(\lambda-1)\left(\beta-\frac{n+2}{2}\right)} e^{(1-\lambda)|x|^{2} /(4 t)}}{(4 \pi)^{(\lambda-1) n / 2} \Gamma(\alpha+\beta)^{\lambda}} \\
& \leq A^{\lambda-1} \frac{\Gamma(\beta)}{(4 \pi)^{(\lambda-1) n / 2} \Gamma(\alpha+\beta)^{\lambda}}=1
\end{aligned}
$$

by (5.30), (5.31), and (5.33). The proof of Theorem 5.4 is now complete.

Since any solution of the approximate $J_{\alpha}$ problem is, after scaling, also a solution of the super and sub $J_{\alpha}$ problems, it follows from Theorems 3.1 and 3.3 that if $\lambda, \alpha$, and $p$ satisfy the conditions in Theorem 3.2 and $(\lambda, \alpha) \in A \cup B \cup C \cup D$ then there do not exist positive constants $C_{1}$ and $C_{2}$ such that the approximate $J_{\alpha}$ problem has a nontrivial nonnegative solution $f \in X^{p}$. Hence Theorem 3.2 follows from the following theorem.

Theorem 5.5. Suppose the constants $\lambda$ and $\alpha$ satisfy

$$
\lambda>1 \quad \text { and } \quad 0<\alpha<\frac{n+2}{2}\left(1-\frac{1}{\lambda}\right) .
$$

Define $f: \mathbb{R}^{n} \times \mathbb{R} \rightarrow[0, \infty)$ by

$$
f(x, t)= \begin{cases}t^{-\frac{\alpha \lambda}{\lambda-1}} w(x / \sqrt{t}) & \text { for }(x, t) \in \mathbb{R}^{n} \times(0, \infty) \\ 0 & \text { for }(x, t) \in \mathbb{R}^{n} \times(-\infty, 0]\end{cases}
$$

where

$$
w(z)=e^{-\frac{\lambda|z|^{2}}{4}}\left(|z|^{2}+1\right)^{-\lambda\left(\frac{n+2}{2}-\frac{\alpha \lambda}{\lambda-1}\right)} \quad \text { for } z \in \mathbb{R}^{n} .
$$

Then

$$
f \in C^{\infty}\left(\mathbb{R}^{n} \times \mathbb{R} \backslash\{(0,0)\}\right) \cap X^{1}
$$

and $f$ is a solution of the approximate $J_{\alpha}$ problem (3.3), (3.4) for some positive constants $C_{1}$ and $C_{2}$ depending only on $n, \lambda$, and $\alpha$.

Moreover if $p \in[1, \infty)$ then $f \in X^{p}$ if and only if

$$
\alpha<\frac{n+2}{2 p}\left(1-\frac{1}{\lambda}\right) .
$$


Proof. We first prove the last sentence of the theorem. Let $p \in[1, \infty)$. then for all $t>0$ we find under the change of variables $x=\sqrt{\tau} z$ that

$$
\begin{aligned}
\iint_{\mathbb{R}^{n} \times(-\infty, t)} f(x, \tau)^{p} d x d \tau & =\int_{0}^{t} \tau^{-\frac{\alpha \lambda p}{\lambda-1}}\left(\int_{\mathbb{R}^{n}} w\left(\frac{x}{\sqrt{\tau}}\right)^{p} d x\right) d \tau \\
& =\int_{0}^{t} \tau^{\frac{n}{2}-\frac{\alpha \lambda p}{\lambda-1}}\left(\int_{\mathbb{R}^{n}} w(z)^{p} d z\right) d \tau
\end{aligned}
$$

Hence, since $\int_{\mathbb{R}^{n}} w(z)^{p} d z<\infty$, we see that $f \in X^{p}$ if and only if

$$
\frac{n}{2}-\frac{\alpha \lambda p}{\lambda-1}>-1
$$

which is equivalent to (5.38).

It follows from (5.34) and the last sentence of the theorem that $f \in X^{1}$. One easily checks that $f \in C^{\infty}\left(\mathbb{R}^{n} \times \mathbb{R} \backslash\{(0,0)\}\right)$. Hence $f$ satisfies (5.37).

We now complete the proof of the theorem by proving that $f$ satisfies the inequalities (3.3). Let $(x, t) \in \mathbb{R}^{n} \times(0, \infty)$ and let $\bar{x}=x / \sqrt{t}$. Then

$$
f(x, t)=t^{-\frac{\alpha \lambda}{\lambda-1}} w(\bar{x})
$$

and under the variables $\tau=t \bar{\tau}$ and $\xi=\sqrt{t} \bar{\xi}$ we get

$$
\Phi_{\alpha}(x-\xi, t-\tau)=t^{\alpha-1-n / 2} \Phi_{\alpha}(\bar{x}-\bar{\xi}, 1-\bar{\tau}) .
$$

Thus

$$
\begin{aligned}
J_{\alpha} f(x, t) & =\int_{0}^{t} \int_{\mathbb{R}^{n}} \Phi_{\alpha}(x-\xi, t-\tau) \tau^{-\frac{\alpha \lambda}{\lambda-1}} w(\xi / \sqrt{\tau}) d \xi d \tau \\
& =t^{\alpha-\frac{\alpha \lambda}{\lambda-1}} \int_{0}^{1} \int_{\mathbb{R}^{n}} \Phi_{\alpha}(\bar{x}-\bar{\xi}, 1-\bar{\tau}) \bar{\tau}^{-\frac{\alpha \lambda}{\lambda-1}} w(\bar{\xi} / \sqrt{\bar{\tau}}) d \bar{\xi} d \bar{\tau} \\
& =t^{-\frac{\alpha}{\lambda-1}} J_{\alpha} f(\bar{x}, 1) .
\end{aligned}
$$

Hence letting

$$
I(x)=\Gamma(\alpha)(4 \pi)^{n / 2} J_{\alpha} f(x, 1)
$$

we obtain from (5.39) that

$$
\frac{J_{\alpha} f(x, t)}{f(x, t)^{1 / \lambda}}=\frac{J_{\alpha} f(\bar{x}, 1)}{w(\bar{x})^{1 / \lambda}}=\frac{I(\bar{x})}{\Gamma(\alpha)(4 \pi)^{n / 2} w(\bar{x})^{1 / \lambda}}
$$

for $(x, t) \in \mathbb{R}^{n} \times(0, \infty)$. Thus, since (3.3) clearly holds in $\mathbb{R}^{n} \times(-\infty, 0]$, in order to prove (3.3) it suffices to prove

$$
0<C_{1} \leq \frac{I(x)}{w(x)^{1 / \lambda}} \leq C_{2} \quad \text { for } x \in \mathbb{R}^{n}
$$

where $C_{1}$ and $C_{2}$ depend only on $n, \lambda$, and $\alpha$ and from (5.40)

$$
I(x)=\int_{0}^{1}(1-\tau)^{\alpha-1-n / 2} \tau^{-\frac{\alpha \lambda}{\lambda-1}} \int_{\mathbb{R}^{n}} e^{-\frac{|x-\xi|^{2}}{4(1-\tau)}} w(\xi / \sqrt{\tau}) d \xi d \tau .
$$


To do this we will need the identity

$$
\left(g_{a} * g_{b}\right)(x)=\left(\frac{\pi a b}{a+b}\right)^{n / 2} g_{a+b}(x) \quad \text { for } x \in \mathbb{R}^{n} \text { and } a, b>0,
$$

where $g_{a}: \mathbb{R}^{n} \rightarrow(0, \infty)$ is defined by

$$
g_{a}(x)=e^{-|x|^{2} / a}
$$

This identity can be proved in a straightforward way using the convolution theorem for the Fourier transform and the well-known transform

$$
\hat{g}_{a}(y)=(\pi a)^{n / 2} e^{-\frac{a|y|^{2}}{4}}
$$

to show that the left and right sides of (5.43) have the same Fourier transform.

We first prove the upper bound in (5.41). Since (5.34) implies

$$
\delta:=\frac{n+2}{2}-\frac{\alpha \lambda}{\lambda-1}>0
$$

it follows from (5.36) that

$$
w(z) \leq e^{-\frac{\lambda|z|^{2}}{4}} \quad \text { for } z \in \mathbb{R}^{n} .
$$

Hence for $x \in \mathbb{R}^{n}$ we obtain from (5.42) that

$$
I(x) \leq \int_{0}^{1}(1-\tau)^{\alpha-1-n / 2} \tau^{-\frac{\alpha \lambda}{\lambda-1}} h_{\tau}(x) d \tau
$$

where

$$
h_{\tau}(x)=\int_{\mathbb{R}^{n}} e^{-\frac{|x-\xi|^{2}}{4(1-\tau)}} e^{-\frac{\lambda|\xi|^{2}}{4 \tau}} d \xi
$$

Defining

$$
\sigma:=1-1 / \lambda \in(0,1)
$$

by (5.34) and using (5.43) we find that

$$
\begin{aligned}
h_{\tau}(x) & =\left(\frac{4 \pi(1-\tau) \tau}{\lambda(1-\sigma \tau)}\right)^{n / 2} e^{-\frac{|x|^{2}}{4(1-\sigma \tau)}} \\
& \leq(4 \pi(1-\tau) \tau)^{n / 2} e^{-\frac{|x|^{2}}{4(1-\sigma \tau)}} \quad \text { for } 0<\tau<1 \text { and } x \in \mathbb{R}^{n} .
\end{aligned}
$$

Thus

$$
I(x) \leq C \int_{0}^{1}(1-\tau)^{\alpha-1} \tau^{\delta-1} e^{-\frac{|x|^{2}}{4(1-\sigma \tau)}} d \tau,
$$

where $\delta$ is defined in (5.44).

Case I. Suppose $|x| \leq 1$. Then by (5.46), (5.45), (5.44), and (5.36),

$$
I(x) \leq C \int_{0}^{1}(1-\tau)^{\alpha-1} \tau^{\delta-1} d \tau=C \leq C w(x)^{1 / \lambda} .
$$


That is the upper bound in (5.41) holds.

Case II. Suppose $|x|>1$. Then by (5.46), (5.45), and (5.44) we have

$$
\begin{aligned}
e^{|x|^{2} / 4} I(x) & \leq C \int_{0}^{1}(1-\tau)^{\alpha-1} \tau^{\delta-1} e^{-\left(\frac{1}{1-\sigma \tau}-1\right) \frac{|x|^{2}}{4}} d \tau \\
& \leq C \int_{0}^{1}(1-\tau)^{\alpha-1} \tau^{\delta-1} e^{-b \tau} d \tau \quad \text { where } b:=\frac{\sigma|x|^{2}}{4}>\frac{\sigma}{4}>0 \\
& \leq C \int_{0}^{b}\left(1-\frac{s}{b}\right)^{\alpha-1}\left(\frac{s}{b}\right)^{\delta-1} e^{-s} \frac{1}{b} d s \quad \text { where } s=b \tau \\
& =C b^{-(\alpha+\delta-1)}\left(I_{1}(b)+I_{2}(b)\right)
\end{aligned}
$$

where

$$
\begin{aligned}
I_{1}(b): & =\int_{0}^{b / 2}(b-s)^{\alpha-1} s^{\delta-1} e^{-s} d s \\
& \leq C b^{\alpha-1} \int_{0}^{b / 2} s^{\delta-1} e^{-s} d s \leq C b^{\alpha-1}
\end{aligned}
$$

and

$$
\begin{aligned}
I_{2}(b): & =\int_{b / 2}^{b}(b-s)^{\alpha-1} s^{\delta-1} e^{-s} d s \\
& \leq e^{-b / 2} \int_{0}^{b}(b-s)^{\alpha-1} s^{\delta-1} d s \\
& =e^{-b / 2} b^{\alpha+\delta-1} \int_{0}^{1}(1-\tau)^{\alpha-1} \tau^{\delta-1} d \tau \quad \text { where } s=b \tau \\
& =C\left(e^{-b / 2} b^{\delta}\right) b^{\alpha-1} \leq C b^{\alpha-1} .
\end{aligned}
$$

Hence from (5.44) we obtain

$$
e^{|x|^{2} / 4} I(x) \leq C b^{-\delta}=C\left(|x|^{2}\right)^{-\left(\frac{n+2}{2}-\frac{\alpha \lambda}{\lambda-1}\right)} .
$$

Thus the upper bound in (5.41) holds when $|x|>1$.

To complete the proof of the theorem we now prove the lower bound in (5.41). Since $w$ is a positive continuous function on $\mathbb{R}^{n}$ we find from (5.42) and for $x \in \mathbb{R}^{n}$ that

$$
I(x) \geq C \int_{0}^{1}(1-\tau)^{\alpha-1-n / 2} \tau^{-\frac{\alpha \lambda}{\lambda-1}} \int_{|\xi|<\sqrt{\tau}} e^{-\frac{|x-\xi|^{2}}{4(1-\tau)}} d \xi d \tau
$$

and thus from (5.44) and for $|x| \leq 2$ we have

$$
\begin{aligned}
I(x) & \geq C \int_{0}^{1}(1-\tau)^{\alpha-1-n / 2} \tau^{\delta-1} e^{-\frac{9}{4(1-\tau)}} d \tau \\
& =C \geq C w(x)^{1 / \lambda} .
\end{aligned}
$$

Hence it remains only to prove the lower bound in (5.41) when

$$
|x|>2 \text {. }
$$


Since for $x \in \mathbb{R}^{n}$ and $\tau>0$, the expression

$$
\frac{\left|B_{|x|}(x) \cap B_{\sqrt{\tau}}(0)\right|}{\left|B_{\sqrt{\tau}}(0)\right|}=: V\left(\frac{|x|}{\sqrt{\tau}}\right)
$$

is an increasing function of $\frac{|x|}{\sqrt{\tau}}$ we have

$$
V\left(\frac{|x|}{\sqrt{\tau}}\right) \geq V(1)=\frac{\left|B_{1}(e) \cap B_{1}(0)\right|}{\left|B_{1}(0)\right|}>0 \quad \text { for } 0<\sqrt{\tau}<|x|
$$

where $e:=(1,0, \ldots 0) \in \mathbb{R}^{n}$. It follows therefore from (5.47) and (5.48) that

$$
\begin{aligned}
I(x) & \geq C \int_{0}^{1}(1-\tau)^{\alpha-1-n / 2} \tau^{-\frac{\alpha \lambda}{\lambda-1}} \int_{\xi \in B_{|x|}(x) \cap B_{\sqrt{\tau}}(0)} e^{-\frac{|x|^{2}}{4(1-\tau)}} d \xi d \tau \\
& \geq C \int_{0}^{1}(1-\tau)^{-\mu} \tau^{\delta-1} e^{-\frac{a}{1-\tau}} d \tau
\end{aligned}
$$

where $\delta$ is defined in (5.44),

$$
\mu:=\frac{n+2}{2}-\alpha, \quad \text { and } \quad a:=\frac{|x|^{2}}{4}>1
$$

by $(5.48)$

Next making the change of variables $s+a=a /(1-\tau)$ in (5.49) and using (5.50) we obtain

$$
\begin{aligned}
I(x) & \geq C \int_{0}^{\infty}\left(\frac{a}{s+a}\right)^{-\mu}\left(\frac{s}{s+a}\right)^{\delta-1} e^{-(s+a)} \frac{a}{(s+a)^{2}} d s \\
& =C a^{1-\mu} e^{-a} \int_{0}^{\infty}(s+a)^{\mu-\delta-1} s^{\delta-1} e^{-s} d s \\
& =C a^{-\delta} e^{-a} \int_{0}^{\infty}\left(1+\frac{s}{a}\right)^{\mu-\delta-1} s^{\delta-1} e^{-s} d s \\
& \geq C e^{-\frac{|x|^{2}}{4}}\left(|x|^{2}\right)^{-\left(\frac{n+2}{2}-\frac{\alpha \lambda}{\lambda-1}\right)} \\
& \geq C w(x)^{1 / \lambda}
\end{aligned}
$$

because

$$
1<1+\frac{s}{a}<1+s \text { for } s>0
$$

by (5.50). Thus the lower bound in (5.41) holds when $|x|>2$.

\section{References}

[1] B. Abdellaoui, A. Attar, R. Bentifour, I. Peral, On fractional p-Laplacian parabolic problem with general data, Ann. Mat. Pura Appl. (4) 197 (2018) 329-356.

[2] J. Aguirre, M. Escobedo, A Cauchy problem for $u_{t}-\Delta u=u^{p}$ with $0<p<1$. Asymptotic behaviour of solutions. Ann. Fac. Sci. Toulouse Math. 8 (1986/87) 175-203.

[3] E. Affili, E. Valdinoci, Decay estimates for evolution equations with classical and fractional time-derivatives, J. Differential Equations 266 (2019) 4027-4060. 
[4] B. Abdellaoui, M. Medina, I. Peral, A. Primo, Optimal results for the fractional heat equation involving the Hardy potential, Nonlinear Anal. 140 (2016) 166-207.

[5] M. Allen, A nondivergence parabolic problem with a fractional time derivative, Differential Integral Equations 31 (2018) 215-230.

[6] M. Allen, L. Caffarelli, A. Vasseur, A parabolic problem with a fractional time derivative, Arch. Ration. Mech. Anal. 221 (2016) 603-630.

[7] I. Athanasopoulos, L. Caffarelli, E. Milakis, On the regularity of the non-dynamic parabolic fractional obstacle problem, J. Differential Equations 265 (2018) 2614-2647.

[8] M. Bonforte, J. L. Vázquez, A priori estimates for fractional nonlinear degenerate diffusion equations on bounded domains, Arch. Ration. Mech. Anal. 218 (2015) 317-362.

[9] H. Chen, L. Véron, Y. Wang, Fractional heat equations with subcritical absorption having a measure as initial data, Nonlinear Anal. 137 (2016) 306-337.

[10] M. G. Delgadino, S. Smith, Hölder estimates for fractional parabolic equations with critical divergence free drifts, Ann. Inst. H. Poincar Anal. Non Linaire 35 (2018) 577-604.

[11] S. Dipierro, E. Valdinoci, V. Vespri, Decay estimates for evolutionary equations with fractional time-diffusion, J. Evol. Equ. 19 (2019) 435-462.

[12] M. Escobedo, M. A. Herrero, Boundedness and blow up for a semilinear reaction-diffusion system, J. Differential Equations 89 (1991) 176-202.

[13] H. Fujita, On the blowing up of solutions of the Cauchy problem for $u_{t}=\Delta u+u^{1+\alpha}$, J. Fac. Sci. Univ. Tokyo Sect. I, 13 (1966), 109-124.

[14] G. Furioli, T. Kawakami, B. Ruf, E. Terraneo, Asymptotic behavior and decay estimates of the solutions for a nonlinear parabolic equation with exponential nonlinearity, J. Differential Equations 262 (2017) 145-180.

[15] C. G. Gal, M. Warma, On some degenerate non-local parabolic equation associated with the fractional p-Laplacian, Dyn. Partial Differ. Equ. 14 (2017) 47-77.

[16] A. Haraux, F. Weissler, Nonuniqueness for a semilinear initial value problem. Indiana Univ. Math. J. 31 (1982) 167-189.

[17] M. Jleli, B. Samet, The decay of mass for a nonlinear fractional reaction-diffusion equation, Math. Methods Appl. Sci. 38 (2015) 1369-1378.

[18] J. Kadlec, Solution of the first boundary value problem for a generalization of the heat equation in classes of functions possessing a fractional derivative with respect to the timevariable, (Russian) Czechoslovak Math. J. 16 (91) (1966) 91-113.

[19] J. Kemppainen, J. Siljander, V. Vergara, R. Zacher, Decay estimates for time-fractional and other non-local in time subdiffusion equations in $\mathbb{R}^{d}$. Math. Ann. 366 (2016) 941-979

[20] M. Mirzazadeh, Analytical study of solitons to nonlinear time fractional parabolic equations. Nonlinear Dynam. 85 (2016) 2569-2576. 
[21] E. Mitidieri, S. I. Pokhozhaev, A priori estimates and the absence of solutions of nonlinear partial differential equations and inequalities. (Russian) Tr. Mat. Inst. Steklova 234 (2001), 1-384; translation in Proc. Steklov Inst. Math. 2001, no. 3(234), 1-362.

[22] L. Molinet, S. Tayachi, Remarks on the Cauchy problem for the one-dimensional quadratic (fractional) heat equation, J. Funct. Anal. 269 (2015) 2305-2327.

[23] K. Nyström, O. Sande, Extension properties and boundary estimates for a fractional heat operator, Nonlinear Anal. 140 (2016) 29-37.

[24] E. Ozbilge, A. Demir, Identification of unknown coefficient in time fractional parabolic equation with mixed boundary conditions via semigroup approach, Dynam. Systems Appl. 24 (2015) 341-348.

[25] F. Punzo, E. Valdinoci, Uniqueness in weighted Lebesgue spaces for a class of fractional parabolic and elliptic equations, J. Differential Equations 258 (2015) 555-587.

[26] P. Quittner, P. Souplet, Superlinear parabolic problems. Blow-up, global existence and steady states, Birkhuser Advanced Texts: Basler Lehrbcher. [Birkhuser Advanced Texts: Basel Textbooks] Birkhuser Verlag, Basel, 2007.

[27] S. G. Samko, Hypersingular integrals and their applications, Analytical Methods and Special Functions, 5, Taylor \& Francis, Ltd., London, 2002.

[28] P. R. Stinga, J. L. Torrea, Regularity theory and extension problem for fractional nonlocal parabolic equations and the master equation, SIAM J. Math. Anal. 49 (2017) 3893-3924.

[29] F. Sun, P. Shi, Global existence and non-existence for a higher-order parabolic equation with time-fractional term, Nonlinear Anal. 75 (2012) 4145-4155.

[30] S. Sugitani, On nonexistence of global solutions for some nonlinear integral equations, Osaka J. Math. 12 (1975) 45-51.

[31] S. Taliaferro, Pointwise bounds and blow-up for nonlinear fractional parabolic inequalities, J. Math. Pures Appl. 133 (2020) 287-328.

[32] V. Varlamov, Long-time asymptotics for the nonlinear heat equation with a fractional Laplacian in a ball, Studia Math. 142 (2000) 71-99.

[33] J. L. Vázquez, B. Volzone, Symmetrization for linear and nonlinear fractional parabolic equations of porous medium type, J. Math. Pures Appl. (9) 101 (2014) 553-582.

[34] J. L. Vázquez, A. de Pablo, F. Quirós, A. Rodríguez, Classical solutions and higher regularity for nonlinear fractional diffusion equations, J. Eur. Math. Soc. 19 (2017) 1949-1975.

[35] V. Vergara, R. Zacher, Optimal decay estimates for time-fractional and other nonlocal subdiffusion equations via energy methods, SIAM J. Math. Anal. 47 (2015) 210-239.

[36] Q-G Zhang, H-R Sun, The blow-up and global existence of solutions of Cauchy problems for a time fractional diffusion equation, Topol. Methods Nonlinear Anal. 46 (2015) 69-92. 